\title{
Characterization of selective layer and biomolecules fouling in polymeric membranes for microalgae filtration applications using 3D FIB/SEM
}

Hélène Roberge ${ }^{1}$, Philippe Moreau ${ }^{2}$, Estelle Couallier ${ }^{3}$ and Patricia Abellan ${ }^{4}$

${ }^{1}$ IMN de Nantes CNRS, Nantes, France, ${ }^{2}$ IMN de Nantes CNRS / Université de Nantes, Nantes, France, ${ }^{3}$, Laboratoire de Génie des Procédés, Environnement et Agroalimentaire, GEPEA - CNRS / Université de Nantes, Saint Nazaire, France, ${ }^{4}$ IMN / University of Nantes, Nantes, France

Membrane filtration processes allow concentrating, separating and purifying the components from a complex mixture in a liquid phase. The two most important properties of a membrane are, thus, its permeability (ability to allow a fluid to pass through it under pressure gradient) and its selectivity (ability to retain a compound). Recently, they have been adapted for microalgae valorization, where filtration employing porous polymer membranes is used to separate and recover lipids and proteins from ground microalgae aqueous extracts. The biomolecules recovered can be used in pharmaceutical industry, cosmetics, food supplements or biofuel industry as biodiesel [1,2]. The permeability and selectivity of the membrane are related to the size and density of its pores, but also to the selective layer thickness (a thin layer placed directly in contact with the fluid to be filtered), as shown by Poiseuille's law [3]. During filtration, the unwanted accumulation of biomolecules at the surface and in the membrane pores, termed fouling, hampers the membrane performances. A detailed characterization of the pore structure, as well as its interaction with the target biomolecules, is essential to understand and help minimize the fouling of the membrane.

Generally, porous polymer materials used as filtration membranes are characterized, based on large-scale indirect measurements and on simulation techniques [4]. Scanning electron microscopy (SEM) coupled with a focused ion beam (FIB) allows characterizing the 3D nanoporous structure of the material with a few nanometers resolution [5]. Data acquisition is particularly challenging with polymer membranes constituted of amorphous materials presenting little contrast and a strong sensitivity to the electron and ion beams [7]. Moreover, 3D reconstructions of porous media present a common issue: the shine through artefact, (material from subsequent slices is imaged through the pores), leading to a pores deformation in the FIB-milling direction [8]. Finally, for lipids and proteins sensitive biomolecules, finding optimal low dose acquisition methods may not be sufficient. Cryo conditions would permit to observe lipids or proteins fouled hydrated membranes while minimizing damage and maintaining material native structure [9].

In this study, we tested two widely used polymer membranes: PAN (polyacrylonitrile, $30 \mathrm{~nm}$, Orelis) and PES $(0.1 \mu \mathrm{m}$ polyethersulfone, Koch), with a nominal pore size of their selective layer of $30 \mathrm{~nm}$ for the PAN and of $100 \mathrm{~nm}$ for the PES. We developed a method to obtain quality 3D reconstructions with a $5 \mathrm{~nm}$ voxel size using 3D FIB/SEM on clean membranes and overcoming beam sensitivity and artefact difficulties. We used segmentation and quantification to obtain experimental properties of PES and PAN filtration membranes. Finally, we used cryo conditions to prepare and observe lipid and protein fouled membranes.

In this presentation, the sample preparation as well as the optimization of acquisition and reconstruction parameters will be described. We will present a method for identifying the selective layer in polymer filtration membranes using FIB/SEM. The quantitative analysis of pore size distribution, porosity, connectivity and tortuosity (figure 1) will be discussed. The properties-structure relationships will be analyzed, by linking these results with the theory of Hagen-Poiseuille on the porous media flow calculation. Our results on both: pristine and fouled membranes, will be presented. 


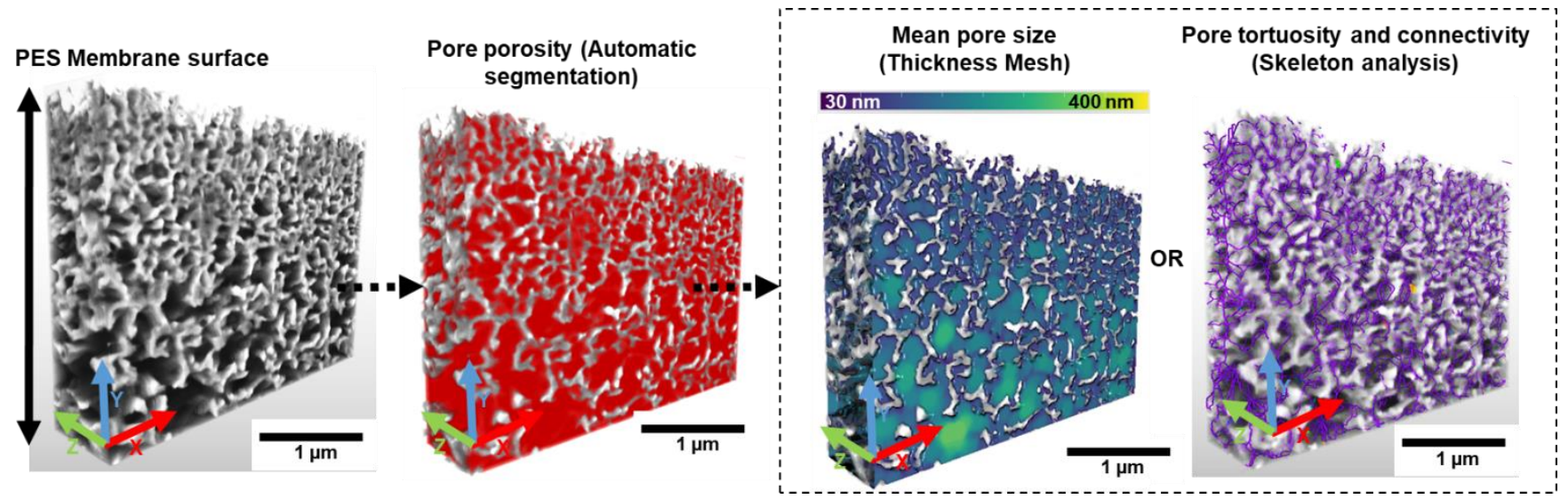

Figure 1. Figure 1 : Illustration of the main 3D analysis steps in the case of the PES membrane. Pores were segmented (DragonFly suite) to extract quantitative information on the volume porosity, the mean pore size (thickness mesh) and the pore tortuosity and connectivity (skeleton).

\section{References}

[1] Rivera et al., Process Biochemistry 89 (2020) 199-207

[2] Villafaña-López et al., Bioresource Technology 288 (2019) , 121539.

[3] AIMAR et al., Techniques de l'Ingénieur, (2010).

[4] Kumar et al., Desalination 318 (2013): 1-8.

[5] Sundaramoorthi et al., Industrial \& Engineering Chemistry Research 55 (2016).

[6] Shi et al., Journal of Membrane Science 487 (2015): 19-31.

[7] Huang et al., Journal of Membrane Science 573 (2019): 7384

[8] Terao et al Journal of Power Sources 347 (2017): 108-13

[9] Kuei et al., Materials Science and Engineering: R: (2020): 100516.

Acknowledgments: The authors would like to thank the financial support by the NExT initiative through national funding by the French National Research Agency (ANR) under the Programme d'investissements d'Avenir (with reference ANR-16-IDEX-0007). The e-BRIDGE project also receives financial support from the Pays de la Loire Région and Nantes Métropole. 